\title{
Research on Rubber Spring Model of High-speed EMU Based on Non-hyperelastic Forces
}

\author{
Chuanbo XU*,**, Maoru CHI*, Liangcheng DAI*, Yiping JIANG*, Yongfa CHEN*, \\ Zhaotuan GUO* \\ *State Key Laboratory of Traction Power, Southwest Jiaotong University, Chengdu, 610031, China, \\ E-mail: cmr2000@163.com (Corresponding author) \\ **Henan Engineering Research Center of Rail Transtit Intelligent Security, Zhengzhou Railway Vocationa \& Technical \\ College, Zhengzhou, 450002, China \\ crossref http://dx.doi.org/10.5755/j02.mech.25630
}

\section{Introduction}

Rubber is a viscoelastic material, it has the function of buffering and absorbing vibration, so in order to reduce the vibration and noise of the train, a large number of rubber components are used in the railway vehicle equipment. With the improvement of safety and comfort of train, the research on rubber components becomes more and more important in the field of vehicle dynamics. The stiffness and damping of rubber components have an important influence on the dynamic performance of the train, so the accurate calculation and prediction of these parameters has always been one of the research hotspots of rubber components, and the establishment of an effective model of rubber components is the basis for accurate calculation of performance parameters. It is very difficult to establish accurate rubber spring model in vehicle dynamics simulation, in literature $[1,2]$, the modeling techniques and methods were summarized. The mechanical characteristics of rubber spring are highly nonlinear, so it is usually based on some test data to establish mechanical model.

The earliest models to simulate rubber material dynamics were Maxwell and K-V (Kelvin-Voigt) model [3], that is, the series and parallel models of spring and damper, which can describe the mechanical behavior of rubber spring to a certain extent. The introduction of more springs and dampers can build more accurate constitutive models, such as Zener, generalized Maxwell and generalized Kelvin model [3]. For rubber springs, these models make the description of mechanical behavior more accurate, but with the increase of parameters, the identification of parameters becomes more and more difficult. Meram [4] studied the Maxwell model of rubber spring using nonlinear buffer and nonlinear spring. Sun D [5] used spring and damping coefficients related to amplitude and frequency to represent spring and damping forces in the model. Allen P [6] selected a new method to determine the elastic response of the rubber components under small amplitude and different preload, and the relationship between absolute displacement and viscoelasticity, the viscoelastic response is described by nonlinear Maxwell model.

Caputo [7] and Bagely [8] first applied fractional differentiation to viscoelastic materials and established constitutive model, Bagely verifies that the fractional differential constitutive model satisfies the thermodynamic principle, and establishes the theoretical basis of the fractional differential model. Many scholars study the fractional differ- ential model from different aspects. The fractional differential model only needs a few parameters to achieve high accuracy, but the expression of the model in the time domain is more complex, it needs to use transcendental function, and the mathematical calculation is not convenient.

Berg [9, 10] proposed a smooth friction model based on input and output functions. Sjöberg [11 - 13] used fractional order to express the viscoelastic force of rubber spring, and considered the internal friction of rubber components. The composite model of three forces superposition is established, including super elasticity, fractional differentiation and generalized friction. The model can effectively describe the mechanical behavior of rubber components, and the significance of each part is clear, the piecewise function of friction part can well describe the amplitude correlation of rubber characteristics. Shi et al. [14-16] used different combinations of spring, damper, friction and fractional differential modules for modeling and analysis, and frequency and amplitude were mainly considered as influencing factors. Luo [17] proposed a model including three components of elastic force, damping force and Maxwell module, which is used to express the correlation with frequency and amplitude. Its calculation amount is less than the existing rubber spring model using friction or fractional order differential module.

Maxwell, K-V model and their related generalized models do not consider the friction factor, so the hysteretic curve of static condition (quasi-static, the static and quasistatic mentioned below are considered to be the same) cannot be explained. The simple model is not accurate enough to describe the dynamic condition, and too many parameters of the generalized model lead to the difficulty of identification. The calculation of the superposition model is complicated because it contains fractional derivatives and friction modules. In view of the above situation, this paper proposes a simplified and effective non-hyperelastic forces (NHEF) model based on the test data. In the model, the viscous force and friction force are unified as NHEF, and the mathematical expression formula is deduced through theoretical analysis. The model is validated and analyzed by test data, it can not only describe the static NHEF, but also describe the dynamic NHEF in the low frequency condition, and it has a good description of both compression and shear conditions. 


\section{NHEF Model}

\subsection{Calculation of NHEF}

Rubber spring is simple in structure, but it has the function of spring and damping at the same time, which can buffer and absorb vibration energy. Spring force is mainly composed of three parts, including super elastic force, viscous force and internal friction force. It is generally believed that the viscous force is mainly related to the strain rate, and the hyperelastic force and internal friction force are mainly related to the displacement. Therefore, three forces are considered simultaneously in the dynamic condition, and only the hyperelastic force and internal friction force are considered in the static (quasi-static) condition. The model established by Sjöberg et al. is based on the superposition of multiple forces. The more different forces are divided, the more accurate of the model in theory, but more errors may be superposed in calculation, and the calculation process is more complex. According to the theory, both viscous force and friction force can be called NHEF, both of them can cause periodic energy consumption. Therefore, in this paper, the viscous force and friction force are no longer distinguished, both of them are considered as non hyperelastic force. According to the force-displacement hysteretic curve of the rubber spring, the periodic energy consumption of the rubber spring is completely caused by the NHEF. The hyperelastic force has no effect on the periodic energy consumption, but only affects the inclination of the hysteretic curve.

In order to facilitate the theoretical derivation of the model formula, the following idealized assumptions are made:

a) it is assumed that when the rubber spring vibrates near a certain pre-pressure, the stiffness caused by the hyperelastic force will not change (considering the compression condition in sections), that is, it will not change with the vibration process under the same condition;

b) it is assumed that the force-displacement hysteretic curve is symmetrical at the center of $(0,0)$ point.

Let the equivalent stiffness of rubber spring is $K$, the spring force is $F_{\text {total }}$ (including hyperelastic force and NHEF), NHEF is $F_{\text {NHEF }}$, the maximum NHEF is $F_{\text {NHEF max }}$, the loss factor is $\eta$, the lag angle is $\alpha$. The schematic diagram of the defined parameters is shown in Fig. 1, and the parameters are specifically defined as follows:

$$
\begin{aligned}
& K=\frac{F_{\text {max }}-F_{\min }}{x_{\max }-x_{\min }}, \\
& F_{\text {NHEFmax }}=\frac{1}{2}\left(F_{\max , x=0}-F_{\min , x=0}\right), \\
& h=\frac{W}{2 K x_{A}^{2}+\frac{1}{2} W}=\frac{S}{2 K x_{A}^{2}+\frac{1}{2} S}=\frac{c_{d} p \omega x_{A}^{2}}{2 K x_{A}^{2}+\frac{1}{2} c_{d} p \omega x_{A}^{2}}, \\
& \alpha=\arctan (\eta),
\end{aligned}
$$

where: $\omega$ is the vibration angle frequency; $x_{A}$ is the displacement at the maximum amplitude; $W$ is the periodic energy consumption; $S$ is the area enclosed by hysteretic curve; $c_{d}$ is equivalent damping.

When the rubber spring vibrates at a certain frequency and amplitude near a certain pre-pressure, the stiffness caused by the hyperelastic force has little change, and the error caused by using the equivalent stiffness $K$ to express the super elastic stiffness is small, so the NHEF $F_{N H E F}$ can be calculated by the following formula:

$$
F_{\text {NHEF }}=F_{\text {total }}-K x \text {, }
$$

where: $x$ is the displacement of the rubber spring relative to the preloading balance position.

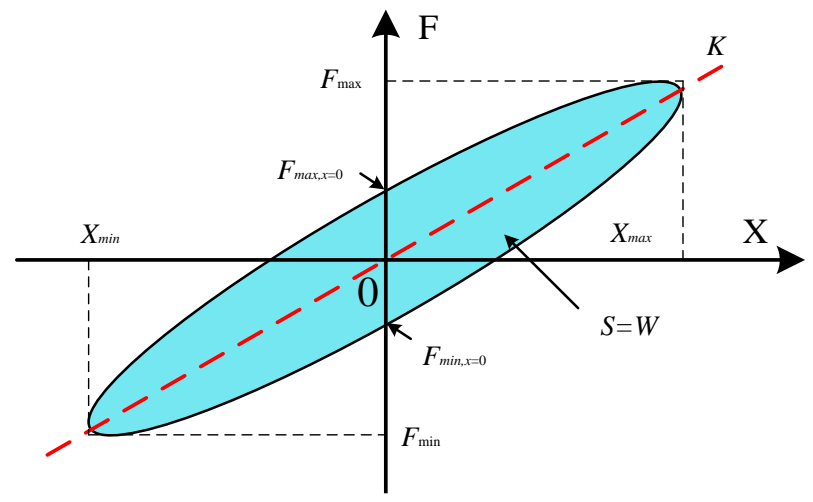

Fig. 1 Diagram of force-displacement hysteretic curve

When the rubber spring vibrates at a certain frequency and amplitude near a certain pre-pressure, the stiffness caused by the hyperelastic force has little change, and the error caused by using the equivalent stiffness $K$ to express the super elastic stiffness is small, so the NHEF $F_{N H E F}$ can be calculated by the following formula:

$$
F_{\text {NHEF }}=F_{\text {total }}-K x \text {, }
$$

where: $x$ is the displacement of the rubber spring relative to the preloading balance position.

In a vibration period of rubber spring, the stiffness of compression test changes more than that of transverse shear test, which is not conducive to the analysis of the law. Therefore, the transverse shear test is used as the research object to derive the model.

Taking the first series rubber pad of a high-speed EMU bogie as an example, the transverse shear test is carried out. Fig. 2 shows the force-displacement hysteresis curve of a rubber spring under the condition of pre-pressure $45 \mathrm{kN}$, frequency $0.5 \mathrm{~Hz}$ and amplitude $A=1 \mathrm{~mm}$. Use Eq. (5) to calculate the NHEF, as shown in Fig. 3, take amplitude $A=1 \mathrm{~mm}$ as horizontal half axis, $F_{\text {NHEFmax }}$ as vertical half axis, and point $(0,0)$ as center to make ellipse in Fig. 3. According to the analysis of Fig. 3, the NHEF-displacement curve is close to a standard ellipse, so we assume that the NHEF-displacement curve is an ellipse, its equation is:

$$
\frac{x^{2}}{A^{2}}+\frac{F_{\text {NHEF }}^{2}}{F_{\text {NHEFmax }}^{2}}=1 \text {. }
$$

According to Eq. (6), the NHEF force can be calculated as follows:

$$
\begin{aligned}
& F_{\text {NHEF }}=\frac{F_{\text {NHEFmax }}}{A} \sqrt{A^{2}-x^{2}} \quad x \uparrow \\
& F_{\text {NHEF }}=-\frac{F_{\text {NHEFmax }}}{A} \sqrt{A^{2}-x^{2}} \quad x \downarrow
\end{aligned}
$$

Therefore, 


$$
\begin{array}{ll}
F_{\text {total }}=K x+\frac{F_{\text {NHEFmax }}}{A} \sqrt{A^{2}-x^{2}} & x \uparrow \\
F_{\text {total }}=K x-\frac{F_{\text {NHEFmax }}}{A} \sqrt{A^{2}-x^{2}} & x \downarrow
\end{array} .
$$

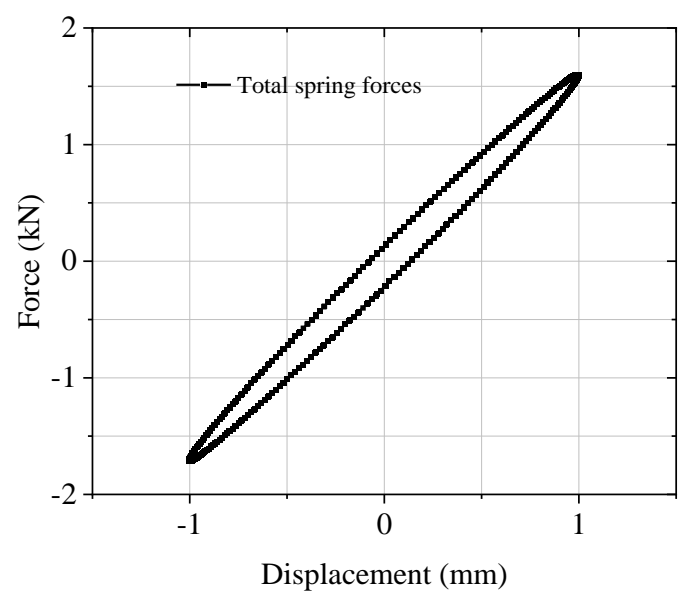

Fig. 2 Force-displacement hysteretic curve under shear condition

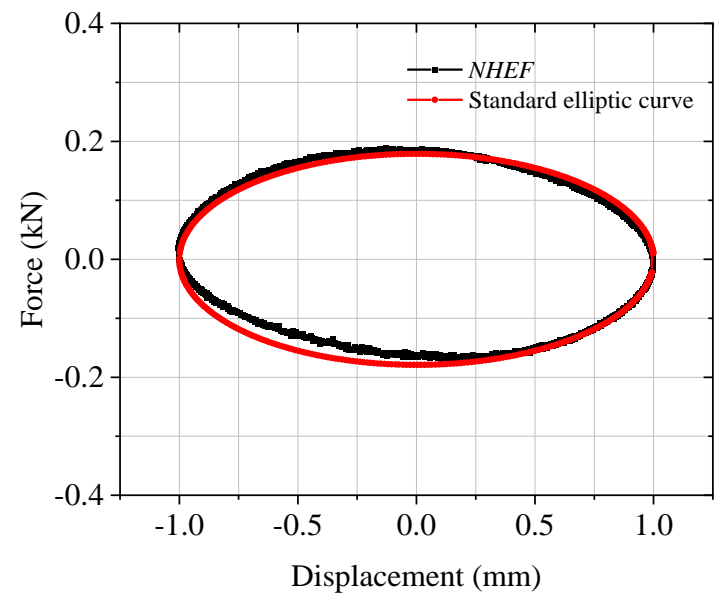

Fig. 3 NHEF- displacement curve under shear condition

2.2. Relationship between NHEF and periodic energy consumption

It can be seen from the theoretical analysis that the NHEF causes the energy consumption of the rubber spring, and the hyperelastic force has no energy consumption in the process of vibration. Therefore, in Fig. 2 and Fig. 3, the area enclosed by the force-displacement curve is the periodic energy consumption of the rubber spring. The NHEF-displacement curve is an approximate ellipse, so the area calculation method of ellipse can be used to estimate the periodic energy consumption of rubber spring. According to that, the following formula can be obtained:

$$
W=S \approx \pi A F_{\text {NHEFmax }} .
$$

It can be seen from Eq. (9), the periodic energy consumption $W$ is only related to the amplitude $A$ and the maximum NHEF $F_{\text {NHEFmax }}$ of the rubber spring under a certain working condition. If it is under the static working condition (quasi-static), the maximum NHEF is the maximum internal friction force.

\section{Test}

\subsection{Test equipment and working condition setting}

In order to further verify the accuracy of Eqs. (7) and (9), a series of annular rubber pads of a high-speed EMU bogie were used for vertical compression and lateral shear tests. Before the rubber pad test, the environment shall be adjusted, the standard temperature shall be set to $23^{\circ} \mathrm{C} \pm 2{ }^{\circ} \mathrm{C}$, and the rubber adjustment time shall not be less than 24 hours. The excitation waveform shall be sine wave, and the preloading position shall be set as the displacement zero point. The static test of vertical compression and transverse shear adopts quasi-static condition, the pre-pressure is $45,57,65$ and $75 \mathrm{kN}$, the average loading time is more than $5 \mathrm{~min}$, and the amplitude is $0.5,1,2$ and $3 \mathrm{~mm}$ for the test. In each working condition, 10 cycles are repeated, and the force-displacement data of the last three cycles are recorded. Before the formal test, apply the maximum vertical load and move for 20 cycles with the maximum vertical displacement, and then start the test after standing for $10 \mathrm{~min}$. During the test, stand for 3 min between each working condition, which is to reduce the influence of stress softening and make the comparison between different working conditions easier. Fig. 4 shows the annular rubber pad used in the test, and Fig. 5 shows the test-bed equipment, which can be used for transverse shear and vertical compression tests.

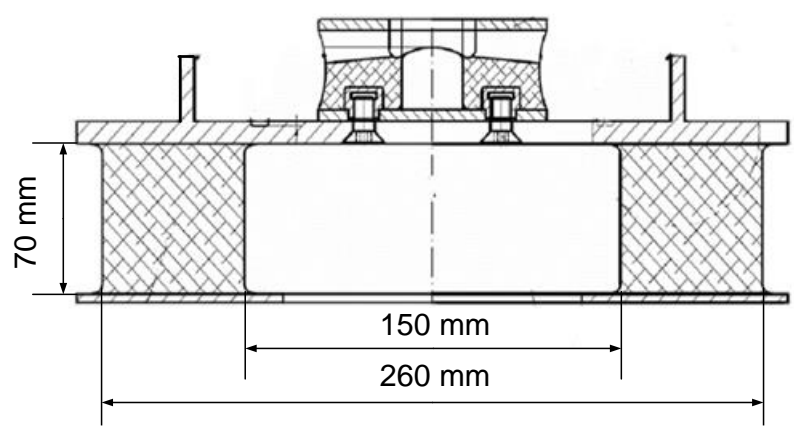

Fig. 4 Annular rubber pad

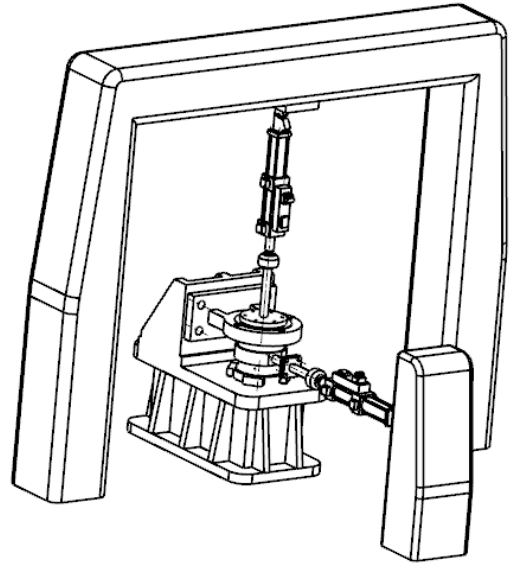

Fig. 5 Test-bed equipment

\subsection{Data processing and parameter identification}

The force-displacement data of each working condition are processed, and the equivalent stiffness is obtained by Eq. (1). Because this paper mainly analyzes the NHEF force, the error of the equivalent stiffness is not analyzed in detail. The calculation result of Eq. (5) is used to replace the 
NHEF value of the test. Because of the small sampling interval, the intersection of the force-displacement hysteretic curve and the $\mathrm{Y}$ axis (displacement zero point) is determined by linear interpolation. The maximum NHEF $F_{\text {NHEFmax }}$ is obtained by Eq. (2). The actual enclosed area of the force-displacement hysteretic curve is obtained by the computer numerical integration. Under the same working condition, the arithmetic mean value is adopted for the data of multiple cycles.

\section{Discussion on NHEF model under shear condition}

\subsection{Error analysis of static shear condition}

From the above analysis, it can see that the NHEF model is suitable for static working conditions. Under static condition, NHEF can be regarded as internal friction force. In the following, the force-displacement curves of the test and the theoretical calculation NHEF are compared under the static condition.

First, the transverse shear case is taken as an example. The pre-pressure is taken as $45 \mathrm{kN}$, the amplitude is $A=1 \mathrm{~mm}$, about $6.25 \mathrm{~min}$ a loading cycle, equivalent to $1 / 375 \mathrm{~Hz}$, which can be considered as quasi-static. According to the test and theoretical calculation results, Fig. 6 is drawn.

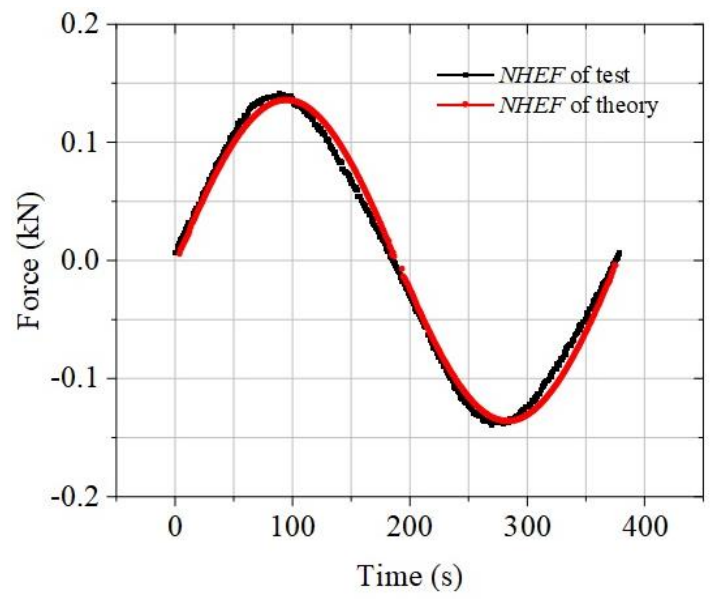

a

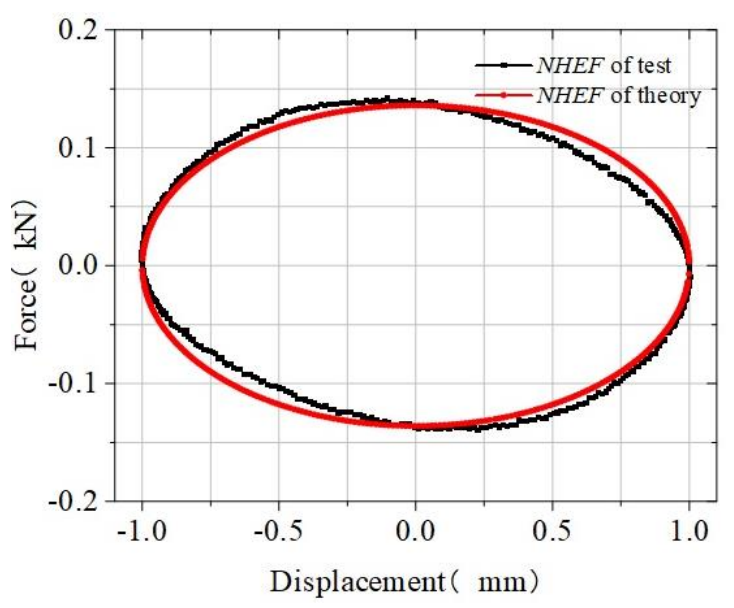

b

Fig. 6 Comparison curve between test and theory value under static shear condition. a) Time-force curve of NHEF ; b) Force-displacement curve of NHEF
It can be seen from Fig. 6 that the theoretical value of NHEF is very close to the calculated value of the test. Fig. 6, a shows a small backward displacement near the peak value of NHEF. In Fig. 6, b, quadrants are divided according to the rectangular coordinate system with $(0,0)$ as the origin, if the absolute value is taken as the comparison object, it can be roughly considered that in the first and third quadrants, the theoretical value of NHEF is less than the test calculation value, in the second and fourth quadrants, the theoretical value is greater than the test value. NHEF mainly affects the energy consumption characteristics of spring. Under quasi-static condition, the proportion of NHEF to the total force of spring is generally within $10 \%$, therefore, NHEF error has little effect on the total force. Because of ideal assumption and calculation method, the absolute error of the total force is the same as that caused by NHEF, and the relative error is very small.

For the convenience of quantitative analysis, the error analysis of NHEF and total spring force is shown in Fig. 7. When making the relative error diagram, a very small amount of data near zero of NHEF is eliminated, because NHEF is very small, the relative error jumps violently, which is related to the test accuracy and measuring equipment, and does not reflect the essential law. Test NHEF is represented by $F_{\text {NHEFtest }}$, theoretical calculation NHEF is represented by $F_{\text {NHEFtheory, absolute error } F_{\text {NHEFerror }} \text { and rela- }}$ tive error $\varepsilon_{N H E F e r r o r}$ are defined as follows:

$$
\begin{aligned}
& F_{\text {NHEFerror }}=F_{\text {NHEFtheory }}-F_{\text {NHEFtest }}, \\
& \varepsilon_{\text {NHEFerror }}=F_{\text {NHEFerror }} / F_{\text {NHEFtest }}
\end{aligned}
$$

According to Fig.7, a, the absolute error of NHEF is the smallest near the balance point (zero displacement), and it is larger near the maximum amplitude. In $x \uparrow$ and $x \downarrow$ stroke, the trend of absolute error is different. By calculation, the maximum absolute error is $-0.0189 \mathrm{kN}$, the average value is $-0.0008 \mathrm{kN}$, the root mean square is $0.0097 \mathrm{kN}$, and the average absolute value of absolute error is $0.0086 \mathrm{kN}$.

According to Fig.7, b, the relative error of NHEF is the smallest near the balance point, and the relative error has a large jump near the maximum amplitude (3\%) because the NHEF is very small. The relative error trends of $x \uparrow$ and $x \downarrow$ stroke are different (distinguish the positive and negative values), and the relative error curve crosses near the balance point, and the overall relative error is within \pm $20 \%$. In order to avoid the influence of the jump value on the calculation results, $3 \%$ of the test points near the maximum amplitude of both ends are removed during the calculation. By calculation, the average value of relative error is $5.4 \%$, the root mean square is $16.06 \%$, and the average absolute value of relative error is $11.99 \%$. As there are still relatively large relative errors of points with small absolute errors, most of the interval errors are actually smaller. The statistical results are obviously affected by some points, but in fact, most of the interval errors are very small.

According to Fig. 7,c, the relative error of the total spring force is small, and it only changes greatly near the balance point, especially near the zero point of the total spring force. By calculation, the maximum relative error is $24.89 \%$, which appears near the zero point of the total spring 
force, but the absolute error is very small, so it does not affect the accuracy of the Eq. (7). The arithmetic mean value is $1.17 \%$, the root mean square is $2.69 \%$, and average absolute value of relative error is $1.55 \%$.

Further analysis from the test results shows that the formula has good accuracy in other quasi-static conditions. In order to make the calculation more intuitive and convenient, the periodic energy consumption Eq. (9) is used for statistical analysis and construct function $\lambda$ :

$$
\lambda=\frac{W}{A F_{\text {NHEFmax }}} .
$$

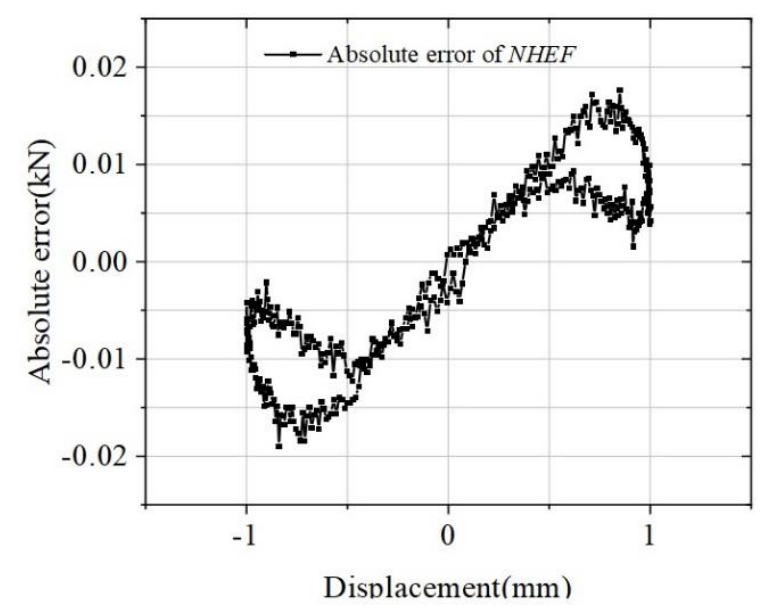

a

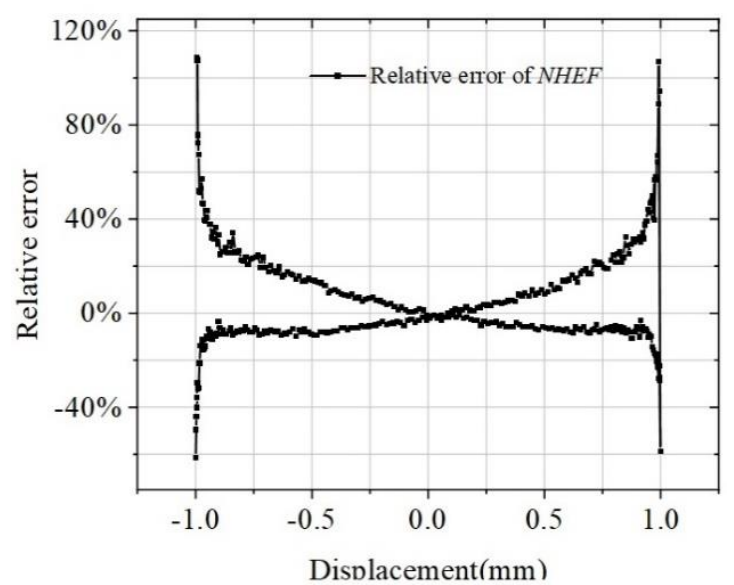

$\mathrm{b}$

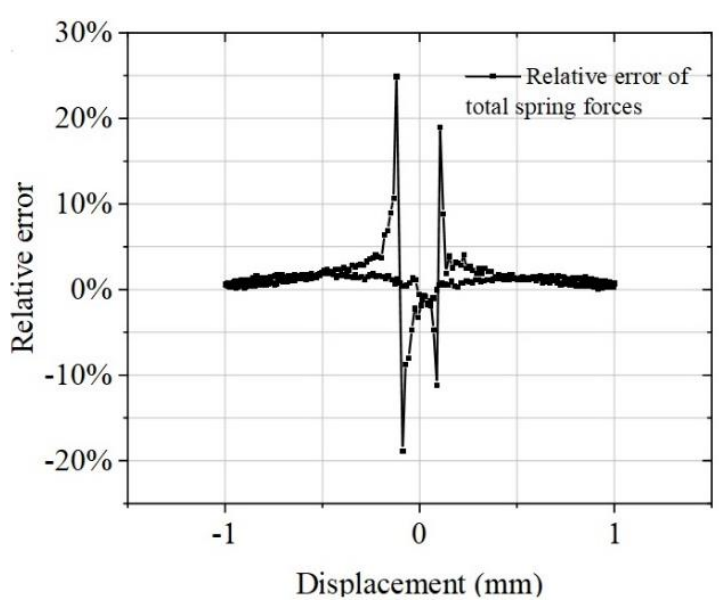

c

Fig. 7 Error analysis diagram of quasi-static shear condition
From the theoretical formula, $\lambda=\pi$ can be obtained. After reasoning, if the value of $\lambda$ calculated from the test data of each working condition is close to $\pi$, it can be considered that the ellipse assumption adopted in the derivation of Eqs. (7) and (9) is reasonable. Table 1 can be obtained by calculating with test data.

Table 1

The $\lambda$ of static shear conditions

\begin{tabular}{|c|c|c|c|c|}
\hline & $0.5 \mathrm{~mm}$ & $1 \mathrm{~mm}$ & $2 \mathrm{~mm}$ & $3 \mathrm{~mm}$ \\
\hline $45 \mathrm{kN}$ & 3.0877 & 3.0810 & 3.0725 & 3.0616 \\
$57 \mathrm{kN}$ & 3.0829 & 3.0800 & 3.0831 & 3.0658 \\
$65 \mathrm{kN}$ & 3.0964 & 3.0875 & 3.0554 & 3.0651 \\
$75 \mathrm{kN}$ & 3.1023 & 3.0946 & 3.0788 & 3.0643 \\
\hline
\end{tabular}

According to Table 1, the average value of $\lambda$ is 3.08, which does not change significantly with the pre-pressure and amplitude. The error of $\lambda$ is only 0.06 , and the relative error is only $1.95 \%$, so it can be considered that the NHEF model is more accurate and effective for the calculation of static shear condition.

\subsection{Error analysis of dynamic shear condition}

The NHEF model considers friction and dynamic viscous force, so it is not only suitable for static condition, but also suitable for dynamic condition. The NHEF analysis is carried out under the condition of pre-compression of $45 \mathrm{kN}$, frequency of $5 \mathrm{~Hz}$ and amplitude of $2 \mathrm{~mm}$. According to the test and theoretical calculation, make comparison curve Fig. 8 and error analysis Fig. 9.

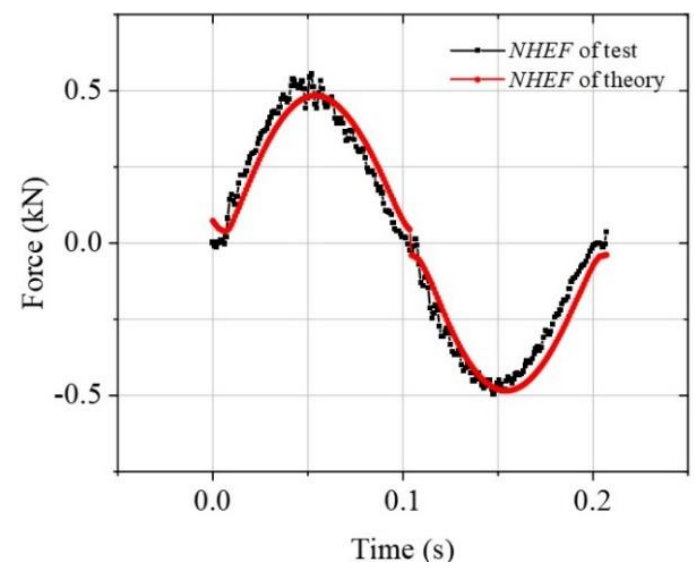

a

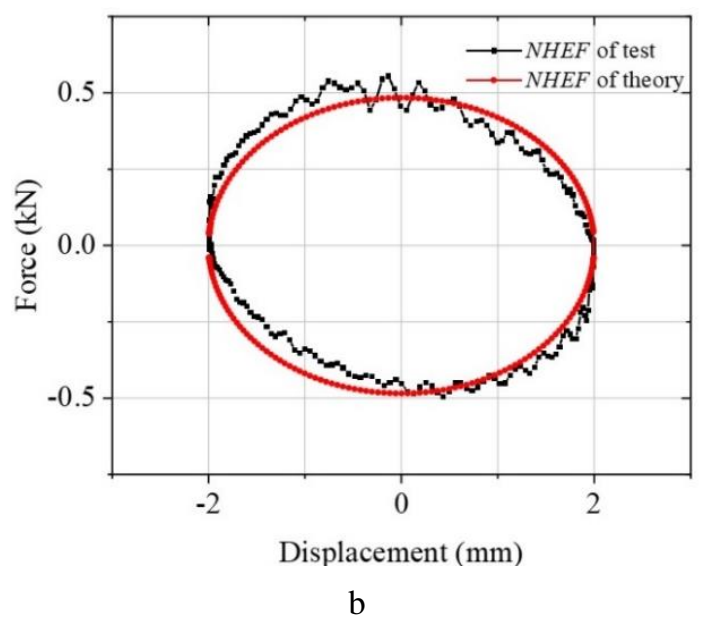

Fig. 8 Comparison curve between test and theory value under dynamic shear condition 
According to Fig. 8, the NHEF theory under the dynamic shear condition can better conform to the test curve, and its characteristics are similar to the quasi-static conditions in Fig. 6, a and Fig. 6, b, only the lag and error amplitude slightly increase.

As shown in Fig. 9, a, when the displacement is far away from the zero point, the absolute error increases accordingly, reaching the maximum value near the maximum amplitude. When the relative error Fig. 9, b is drawn and calculated, the test points near the maximum amplitude (3\%) at both ends are still removed for drawing and statistical calculation. The statistical results show that the mean absolute error of NHEF is $-0.012 \mathrm{kN}$, the mean absolute value of absolute error is $0.0535 \mathrm{kN}$, and the root mean square is $0.0813 \mathrm{kN}$. The mean value of relative error is $7.27 \%$, the mean absolute value is $23.31 \%$, and the root mean square is $32.7 \%$.

Further analysis of the data shows that when the frequency and amplitude increase, the deformation and offset of the rubber spring NHEF curve relative to the ellipse become larger, while the absolute error and relative error increase. Because of the deviation in the positive and negative directions, the mean error is still very small. The error of NHEF model below $5 \mathrm{~Hz}$ is very small and has high calculation accuracy, but above $7 \mathrm{~Hz}$, the calculation accuracy decreases obviously. This is because the assumed error of equivalent hyperelastic stiffness increases with the increase of frequency, that is to say, it is not the error of NHEF model itself, but the problem of experimental data processing used in comparison.

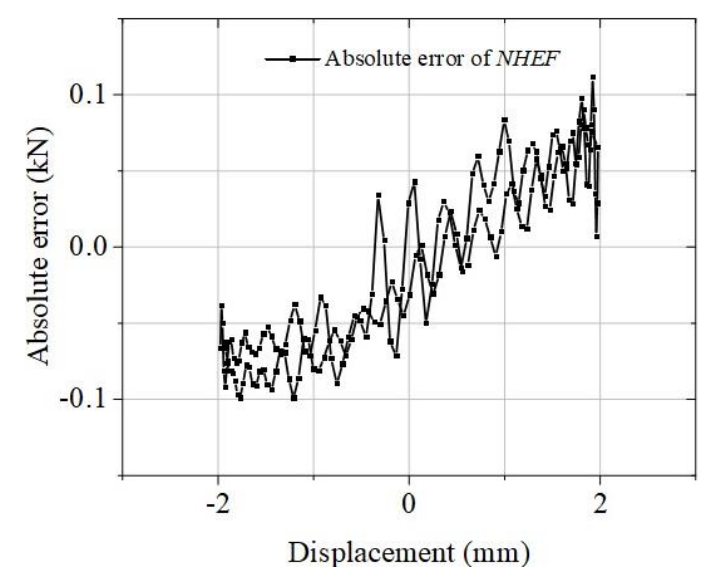

a

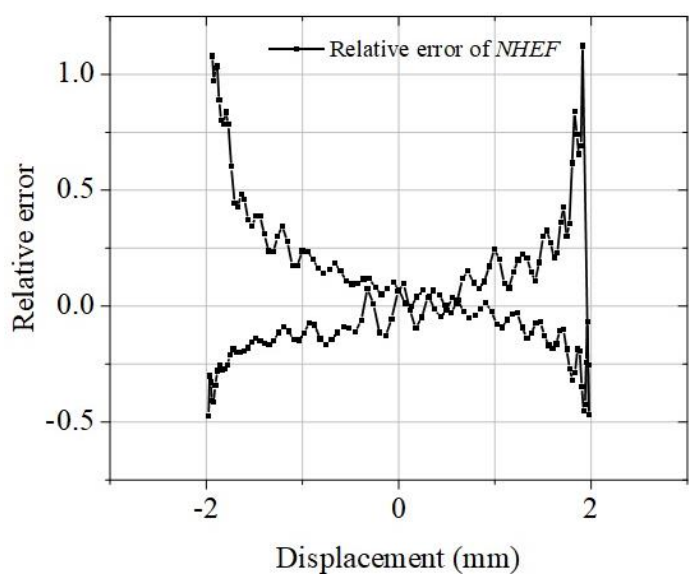

b

Fig. 9 Error analysis diagram of dynamic shear condition
Based on the dynamic shear condition, the accuracy of the periodic energy consumption Eq. (9) is analyzed by using the constructed function $\lambda$, and the calculation results of $\lambda$ are shown in Table 2 .

Table 2

Calculated $\lambda$ values based on dynamic shear test

\begin{tabular}{|c|c|c|c|c|}
\hline \multirow{2}{*}{$0.5 \mathrm{~Hz}$} & 3.1262 & 3.0731 & 3.0707 & 3.0631 \\
\cline { 2 - 5 } $1 \mathrm{~Hz}$ & 3.1409 & 3.0971 & 3.0427 & 3.0793 \\
$2 \mathrm{~Hz}$ & 3.0568 & 3.0203 & 3.1160 & 3.0415 \\
$5 \mathrm{~Hz}$ & 3.0768 & 3.0868 & 3.0722 & 3.1005 \\
$7 \mathrm{~Hz}$ & 3.2607 & 3.2690 & 3.1452 & 3.2028 \\
$10 \mathrm{~Hz}$ & 3.0867 & 3.0648 & 3.1278 & 3.0712 \\
\hline & \multicolumn{4}{|c|}{$57 \mathrm{kN}$} \\
\cline { 2 - 5 } & $0.5 \mathrm{~mm}$ & $1 \mathrm{~mm}$ & $2 \mathrm{~mm}$ & $3 \mathrm{~mm}$ \\
\hline $0.5 \mathrm{~Hz}$ & 3.1343 & 3.1059 & 3.0906 & 3.0986 \\
$1 \mathrm{~Hz}$ & 3.1162 & 3.0902 & 3.0599 & 3.0746 \\
$2 \mathrm{~Hz}$ & 3.0717 & 3.0939 & 3.1005 & 3.0874 \\
$5 \mathrm{~Hz}$ & 3.1382 & 3.0763 & 3.1205 & 3.1245 \\
$7 \mathrm{~Hz}$ & 3.2616 & 3.2263 & 3.1536 & 3.0584 \\
$10 \mathrm{~Hz}$ & 3.1522 & 3.1218 & 3.1960 & 3.1836 \\
\hline \multirow{4}{*}{$65 \mathrm{kN}$} \\
\cline { 2 - 5 } & $0.5 \mathrm{~mm}$ & $1 \mathrm{~mm}$ & $2 \mathrm{~mm}$ & $3 \mathrm{~mm}$ \\
\hline $0.5 \mathrm{~Hz}$ & 3.0771 & 3.0566 & 3.0799 & 3.0746 \\
$1 \mathrm{~Hz}$ & 3.0502 & 3.0898 & 3.1018 & 3.0525 \\
$2 \mathrm{~Hz}$ & 3.1276 & 3.0186 & 3.0541 & 3.0782 \\
$5 \mathrm{~Hz}$ & 3.0992 & 3.1561 & 3.1131 & 3.0732 \\
$7 \mathrm{~Hz}$ & 3.0584 & 3.2379 & 3.1677 & 3.2004 \\
$10 \mathrm{~Hz}$ & 2.9981 & 3.2732 & 3.0307 & 3.1099 \\
\hline & \multicolumn{4}{|c|}{$75 \mathrm{kN}$} \\
\cline { 2 - 5 } & $0.5 \mathrm{~mm}$ & $1 \mathrm{~mm}$ & $2 \mathrm{~mm}$ & $3 \mathrm{~mm}$ \\
\hline $0.5 \mathrm{~Hz}$ & 3.1224 & 3.0573 & 3.0666 & 3.0465 \\
$1 \mathrm{~Hz}$ & 3.0871 & 3.0838 & 3.0800 & 3.0531 \\
$2 \mathrm{~Hz}$ & 3.0490 & 3.0876 & 3.0625 & 3.0706 \\
$5 \mathrm{~Hz}$ & 3.0639 & 3.1937 & 3.2674 & 3.0683 \\
$7 \mathrm{~Hz}$ & 3.1408 & 3.3165 & 3.1739 & 3.1785 \\
$10 \mathrm{~Hz}$ & 2.9187 & 3.1415 & 2.9811 & 3.1310 \\
\hline
\end{tabular}

According to Table 2, the mean value is 3.11, which does not change significantly with the pre-pressure, amplitude and frequency, and the difference between the mean value and the theoretical value is only $0.96 \%$. That is to say, when predicting the periodic energy consumption, the Eq. (9) derived from the NHEF model is quite accurate. When the frequency increases, the calculation error of NHEF model increases, but the calculation of energy consumption is not affected, and the error does not increase with the frequency. This is because the force-displacement curve of NHEF has different error directions in different quadrants, and the mean value of error is always small. Most of the errors in the calculation of the area enclosed by the forcedisplacement curve by Eq. (9) can offset each other, so that the calculation of periodic energy consumption is always more accurate, so that Eq. (9) has a larger application range.

\section{Discussion on NHEF model under compression condi- tion}

\subsection{Error analysis of static compression condition}

For the static compression condition, because the total compression amount of rubber spring is different under different displacement, its vertical stiffness has obvious change, and the greater the excitation amplitude, the more 
obvious the change. As shown in Fig.10, under the compression condition, the force displacement curve of the rubber spring is in the crescent shape with the two ends up warped, and the line between the peak points is above the center point of the figure. From the data analysis, the error of equivalent stiffness $K$ is transferred to the calculation of NHEF test value, referring to Eqs. (1) and (5). The bending of the force displacement curve similar to the crescent is very small, but the proportion of NHEF to the total spring force is also very small, the small error of hyperelastic force transferred to NHEF will be very significant. As shown in Fig. 11, the NHEF curve is seriously deformed.

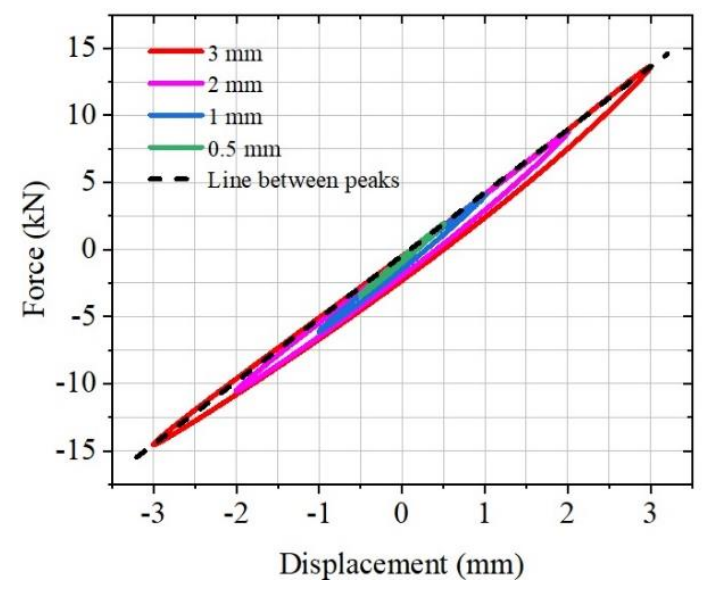

Fig. 10 Total spring force-displacement curve under static compression condition

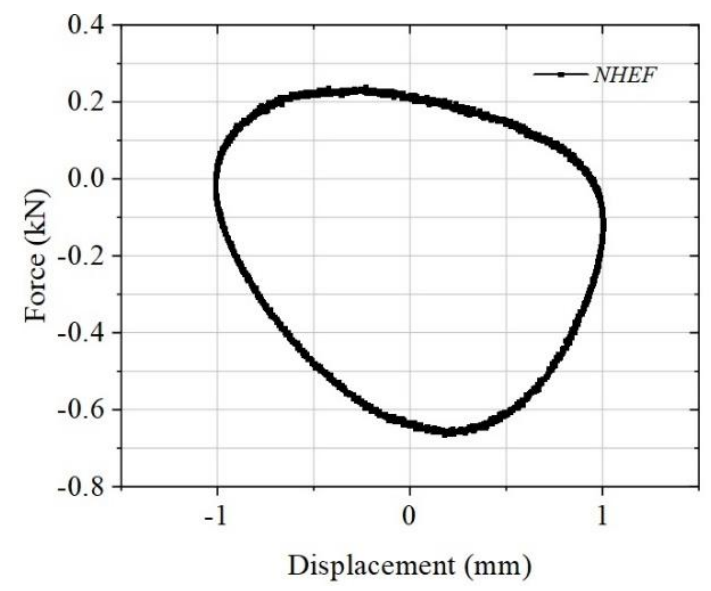

Fig. 11 NHEF-displacement curve for linear stiffness assumption

In order to calculate the NHEF more accurately, the segment equivalent linear stiffness is adopted for each working condition of static compression, with the zero displacement as the dividing point, the equivalent stiffness of the positive displacement part is expressed by $K_{1}$, and the negative displacement part is expressed by $K_{2}$. The definition is as follows:

$$
\begin{array}{ll}
K_{1}=\frac{F_{\text {max }}}{x_{\text {max }}} & x \geq 0 \\
K_{2}=\frac{F_{\text {min }}}{x_{\text {min }}} & x<0
\end{array}
$$

Eq. (5) is modified as follows:

$$
\begin{array}{ll}
F_{\text {NHEF }}=F_{\text {total }}-K_{1} x & x \geq 0 \\
F_{\text {NHEF }}=F_{\text {total }}-K_{2} x & x<0
\end{array}
$$

The quasi-static condition with a pre-pressure of $45 \mathrm{kN}$, an amplitude of $1 \mathrm{~mm}$ and a loading period of $6.25 \mathrm{~min}$ is selected as the analysis object. The value of $F_{\text {NHEFmax }}$ is $0.32659 \mathrm{kN}$. According to Eq. (12), the value of $K_{1}$ is $5.1165 \mathrm{kN} / \mathrm{mm}$, and the $K_{2}$ is $4.985 \mathrm{kN} / \mathrm{mm}$. Test result $F_{N H E F}$ is calculated by Eq. (13), theoretical NHEF is still calculated by Eq. (7), compare and draw Fig. 12.

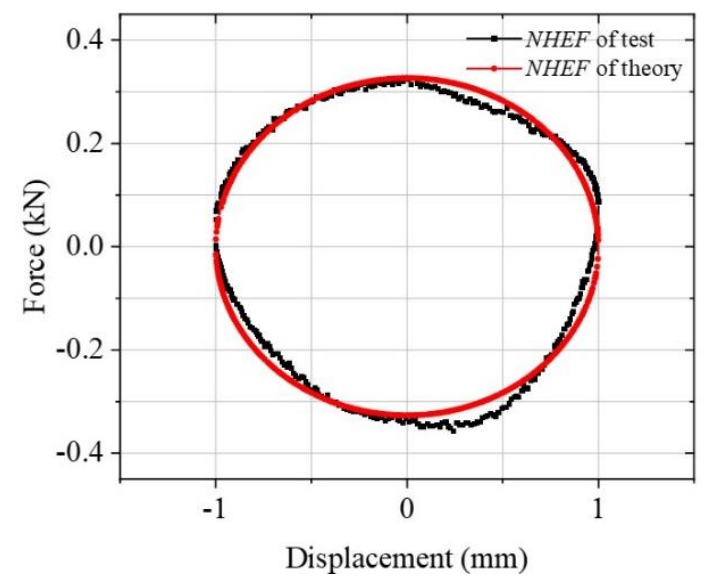

Fig. 12 NHEF-displacement curve under quasi-static compression condition

It can be seen from Fig. 12 that the NHEF model is relatively accurate in describing the test curve, and the error in part of $x>0$ is relatively large, but it is still in a small range. This is because in the $x>0$ part, the nonlinearity of rubber spring stiffness is more obvious, and the error caused by the assumption of equivalent linearization is larger. The error analysis is shown in Fig. 13. In Fig. 13, a, the downward bending of the absolute error image should be caused by piecewise linearization. According to statistical calculation, the average absolute error is $-0.009 \mathrm{kN}$, the average absolute value of absolute error is $0.0225 \mathrm{kN}$, and the root mean square is $0.0283 \mathrm{kN}$. In Fig. 13, b, when calculating the relative error, $3 \%$ points at both ends are still eliminated, the arithmetic mean value is $2.13 \%$, the mean absolute value is $14.91 \%$, and the root mean square is $24.81 \%$.

In order to further verify the accuracy of the model under different working conditions of static compression, the constructed function $\lambda$ is calculated with the test data of each working condition. By calculation, the average value of $\lambda$ is 3.0613 and the root mean square is 3.0625 . The relative error between the calculated value of $\lambda$ and $\pi$ is only $2.61 \%$, and the fluctuation of each working condition is very small. It can be seen that the calculation of the periodic energy consumption of Eq. (9) in the quasi-static compression working condition is relatively accurate, which also shows that the NHEF model is reasonable.

\subsection{Error analysis of dynamic compression condition}

In the discussion of dynamic compression condition, Eq. (13) is also used to calculate the test NHEF. The comparison curve between the test and theoretical value is shown in Fig. 14, and Fig. 15 is drawn according to the error analysis results. From Fig. 14, it can be seen that the test and theoretical NHEF curves are very close on the whole, and 
only there is a large error near the maximum positive amplitude. This is because the nonlinear change of the vertical equivalent stiffness of the spring in this area is obvious, and the sectional equivalent linearization has a large error near the maximum positive amplitude. According to Fig.15, for the absolute error, its average value is $-0.0352 \mathrm{kN}$, the average absolute value is $0.1082 \mathrm{kN}$, and the root mean square is $0.1568 \mathrm{kN}$; for the relative error after eliminating $3 \%$ points at both ends, the average value is $5.04 \%$, the average absolute value is $17.31 \%$, and the root mean square is $26.93 \%$.

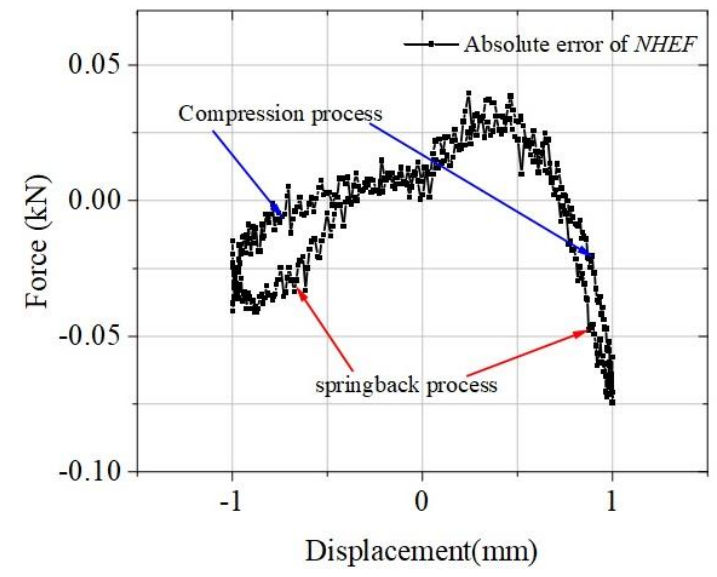

a

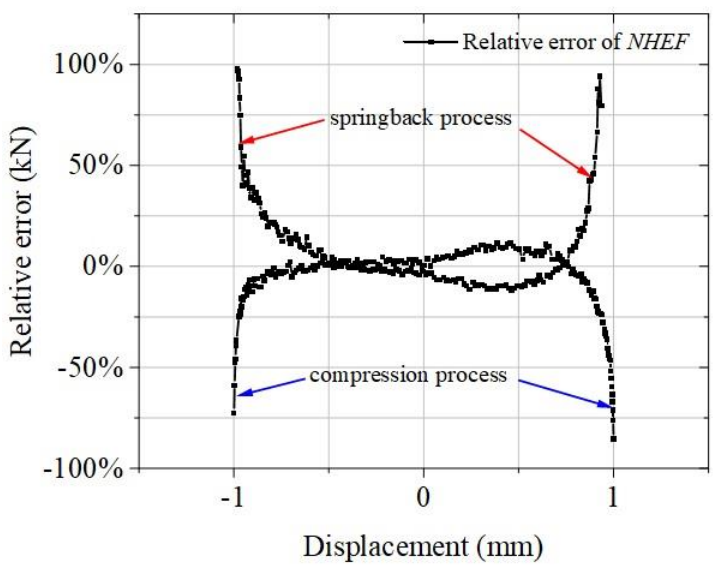

b

Fig. 13 Error analysis diagram of NHEF under quasi-static compression condition

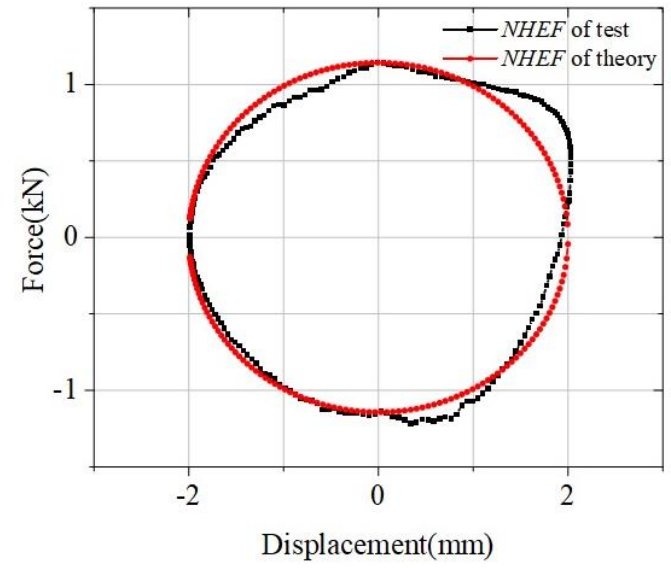

Fig. 14 NHEF-displacement curve under dynamic compression condition
The calculation of construct function $\lambda$ of dynamic compression condition shows that the mean value is 3.146 and the root mean square is 3.1585 , which is almost consistent with the theoretical value $\pi$ and the relative error is within $1 \%$. According to the results, it can be concluded that the NHEF model is quite accurate for the calculation of periodic energy consumption under the dynamic compression conditions.
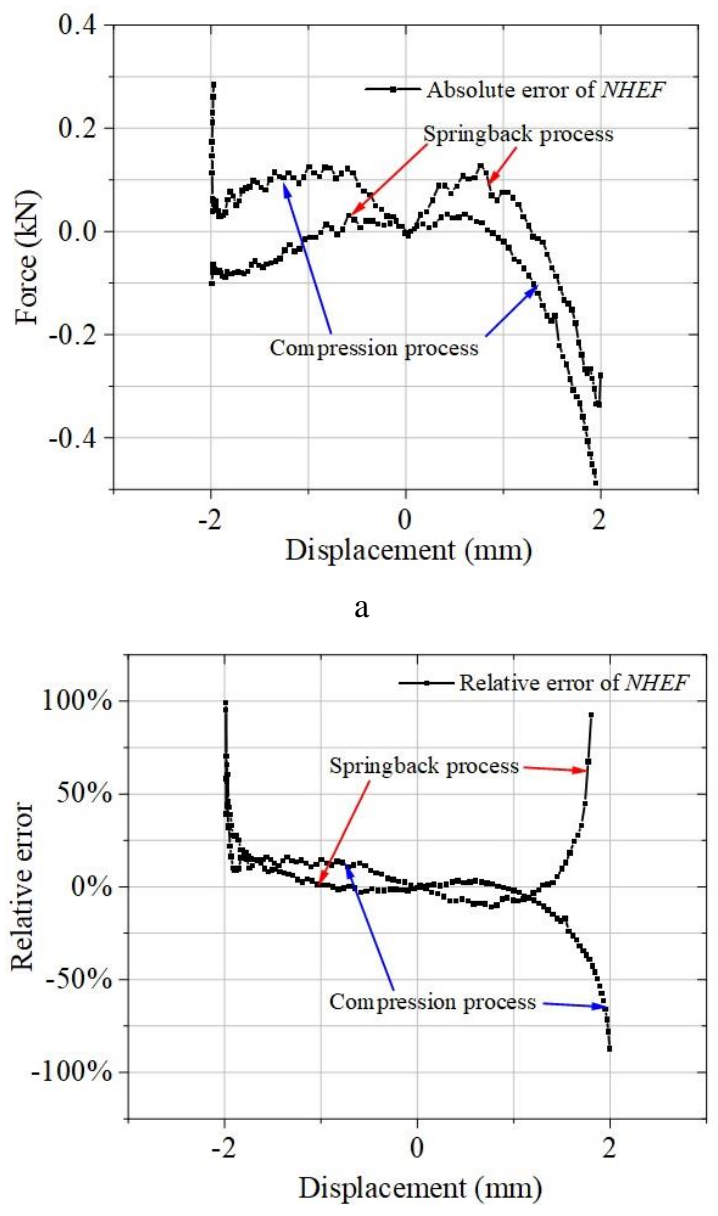

$\mathrm{b}$

Fig. 15 Error analysis diagram of NHEF under dynamic compression condition. a) The curve of absolute error; b) The curve of relative error

Further analysis of the data shows that the theoretical error of the dynamic compression condition is larger than that of the quasi-static compression condition, which is mainly due to the stronger nonlinearity of the vertical dynamic stiffness. With the increase of frequency, the error increases gradually, but when the frequency is not high, the NHEF model has high accuracy. The $\lambda$-function value of dynamic compression condition is analyzed in Fig. 16. From Fig. 16, the $\lambda$ has an increasing trend with the increase of frequency, that is to say, the periodic energy consumption has an increase trend with the increase of frequency. The calculation Eq. (9) of periodic energy consumption has high accuracy under all working conditions, and has the highest calculation accuracy near $5 \mathrm{~Hz}$, because the constructor $\lambda$ calculated from the test data is closest to $\pi$ at $5 \mathrm{~Hz}$. 


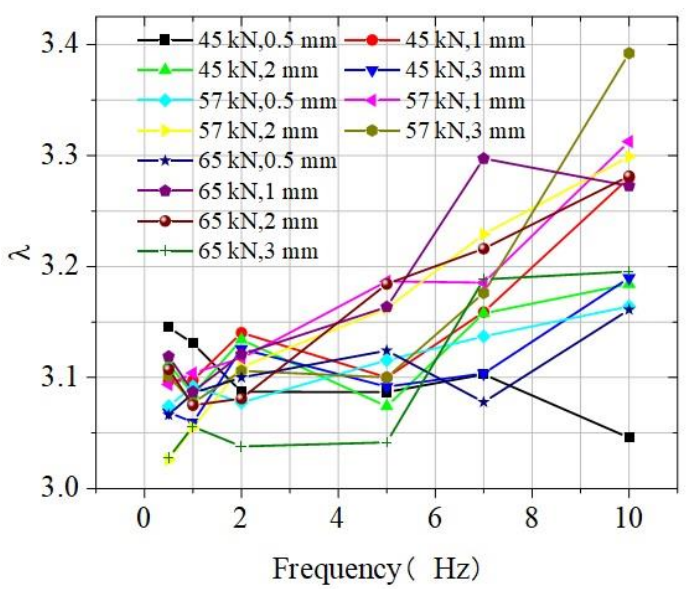

Fig.16 The relationship between $\lambda$ and frequency under compression conditions

\section{Conclusion and future work}

In this paper, the NHEF model formula and periodic energy consumption formula are derived, and the first series rubber pad of high-speed EMU is selected for the test. Through the comparative analysis of the dynamic-static tests under the shear and compression conditions, the following conclusions can be drawn.

1. Under quasi-static and dynamic conditions, the NHEF model can better describe the actual NHEF, the overall error is very small, only there is a large error near the maximum amplitude, so the model can better predict the NHEF of rubber spring.

2. The accuracy of NHEF model in shear condition is better than that in compression condition, mainly because the nonlinear change of equivalent stiffness is more obvious in compression condition, and the calculation error of test NHEF is increased. The error between calculated NHEF and theoretical value is obviously reduced after using segmental equivalent stiffness.

3. The accuracy of NHEF model in quasi-static condition is better than that in dynamic condition. Under the dynamic condition, although the average error is stable, the root mean square error tends to increase after the frequency is greater than $5 \mathrm{~Hz}$, that is to say, the error tends to increase in both positive and negative directions.

4. The calculation formula of periodic energy consumption has a higher calculation accuracy for all test conditions. Because the NHEF two-way error has a counteracting effect when calculating the periodic energy consumption, its application range is larger than that of NHEF model, and the calculation error is stable at a lower value under different conditions.

The calculation of NHEF plays an important role in the study of dynamic characteristics and dynamic temperature rise of rubber spring. It can be seen from the above studies that the NHEF model can better describe the NHEF of static and dynamic low frequencies, but the error tends to increase with the increase of frequency. According to the law of model error, the relationship between lag angle $\alpha$ and model error will be considered in the later research, and the NHEF model will be further modified. In addition, it is necessary to study the nonlinear variation law of the hyperelastic part in a vibration period, so as to establish a complete rubber spring nonlinear model by combining NHEF model.

\section{Funding statement}

This research has been supported by the National Key R\&D Program of China (Grant Nos. 2016YFB120404, Grant Nos.2018YFB1201700), and by the Independent Subject of State Key Laboratory of Traction Power (Grant Nos. 2018TPL_T04).

\section{References}

1. Bruni, S.; Vinolas, J.; Berg, M.; Polach, O.; Stichel, S. 2011. Modelling of suspension components in a rail vehicle dynamics context, Veh Syst Dyn 49(7):102110721.

http://dx.doi.org/10.1080/00423114.2011.586430.

2. Evans, J.; Berg, M. 2009. Challenges in simulation of rail vehicle dynamics, Veh Syst Dyn 47(8):1023-1048. http://dx.doi.org/10.1080/00423110903071674.

3. Montgomery, T. Shaw; William, J. MacKnight. 2005. Introduction to Polymer Viscoelasticity, The third Edition. John Wiley \& Sons, Inc.

4. Meram, A. 2019. Dynamic characterization of elastomer buffer under impact loading by low-velocity drop test method, Polymer Testing 79: article ID 074501. http://dx.doi.org/10.1016/j.polymertesting.2019.106013.

5. Sun, D.; Chen, Z.; Zhang, Z.; Eberhard, P. 2011. Modeling and parameter identification of amplitudeand frequency-dependent rubber isolator, J Cent South Univ T 18(3):672-678. http://dx.doi.org/10.1007/s11771-011-0746-y.

6. Allen, P.; Hameed, A.; Goyder, H. 2007. Measurement and modelling of carbon black filled natural rubber components, Int J Veh Syst Model Test 2(4):315-344. http://dx.doi.org/10.1504/IJVSMT.2007.017118.

7. Caputo, M.; Mainardi, F. 1971. Linear models of dissipation in anelastic solids, Rivista Del Nuovo Cimento 1(2):161-198. http://dx.doi.org/10.1007/BF02820620.

8. Bagley, R. L. 1979. Application of generalized derivates to viscoelasticity, Air Force Institute of Technology, TR-79-4103.

9. Berg, M. 1998. A non-linear rubber spring model for rail vehicle dynamics analysism Veh Syst Dyn 30(3-4): 197212.

http://dx.doi.org/10.1080/00423119808969447.

10. Berg, M. 1997. A model for rubber springs in the dynamic analysis of rail vehicles. I Mech E Part F: J Rail Rapid Transit 211(2):95-108. http://dx.doi.org/10.1243/0954409971530941.

11. Sjöberg, M. 2000. Rubber isolators-measurements and modeling using fractional derivatives and friction, SAE Technical Papers, Truck and Bus Meeting and Exposition. http://dx.doi.org/10.4271/2000-01-3518.

12. Sjöberg, M. 2002. Non-linear behavior of a rubber isolator system using fractional derivatives, Veh Syst Dyn 37(3): 217-236. http://dx.doi.org/10.1076/vesd.37.3.217.3532.

13. Sjöberg, M.; Kari, L. 2003. Nonlinear isolator dynamics at finite deformations: An effective hyperelastic, fractional derivative, generalized friction model, Nonlinear Dyn 33(3): 323-336. http://dx.doi.org/10.1023/A:1026037703124. 
14. Wu, J.; Shangguan, W. 2008. Modeling and applications of dynamic characteristics for rubber isolators using viscoelastic fractional derivative model, Engineering Mechanics 25(1): 161-166 (in Chinese).

15. Shi, H.; Wu, P. 2016. A nonlinear rubber spring model containing fractional derivatives for use in railroad vehicle dynamic analysis, P I Mech Eng F-J Rai 230(7): 1745-1759. http://dx.doi.org/10.1177/0954409715614871.

16. Zhang, D.; Zhu, S. 2017. A fractional derivative model for rubber spring of primary suspension in railway vehicle dynamics, ASCE-ASME Journal of Risk and Uncertainty in Engineering Systems, Part B: Mechanical Engineering 3(3): 030908. http://dx.doi.org/10.1115/1.4036706.

17. Luo, R.; Shi, H.; Guo, J.; Huang, L.; Wang, J. 2019. A nonlinear rubber spring model for the dynamics simulation of a high speed train, Veh Syst Dyn 1:1-18. http://dx.doi.org/10.1080/00423114.2019.1624788
Ch. Xu, M. Chi, L. Dai, Y. Jiang, Y. Chen, Z. Guo

\section{RESEARCH ON RUBBER SPRING MODEL OF HIGH- SPEED EMU BASED ON NON-HYPERELASTIC FORCES}

\section{S u m m a r y}

The research on the mechanical model of rubber spring is one of the hot spots in train dynamics. In order to accurately calculate the viscoelastic force of the rubber spring, especially the non-hyperelastic forces (NHEF) part, a NHEF model is proposed based on the elliptic approximation method. Furthermore, the calculation formula of periodic energy consumption is put forward. The NHEF model is verified by experiments, and the function $\lambda$ isconstructed to verify the formula of periodic energy consumption. The calculation results showed that the NHEF model had high accuracy in predicting the dynamic and quasi-static NHEF of rubber spring, the prediction accuracy of shear condition was better than that of compression condition, and the accuracy of quasi-static condition was better than that of dynamic condition; the calculation formula of periodic energy consumption had a good prediction accuracy in all working conditions.

Keywords: train dynamics, rubber spring, non-hyperelastic forces model, elliptic approximation, periodic energy consumption. 